Results Of the 10 surveys sent out all recipients responded. 2 trusts have access to TNE with only one trust having access to a specific TN service performing approximately 150-200 per year. The 2 trusts with access to TNE had both received training in TNE from industry and also in-house training. The trust with a TNE service had also received training from other endoscopists experienced in TNE and an ENT surgeon. When compared with standard endoscopy $30 \%$ thought views were worse, $60 \%$ the same and $10 \%$ unable to comment (due to lack of experience of TNE). $60 \%$ thought biopsy samples were adequate, $20 \%$ too small and $20 \%$ unable to comment.

Advantages of TNE: 2 felt unable to comment due to lack of familiarity with this method. Improved patient tolerance was the main advantage stated by 7 with improved comfort, less gagging and reduced sedation requirements, with 1 stating less nursing support and therefore potential for evening lists and improving capacity issues as the main advantage.

Disadvantages: 2 unable to comment, 2 no disadvantages, 2stated cost of set up, 1- failure of nasal passage, 1-narrow channel limits therapy, 1-prolonged preparation time compared to throat spray and 1- poor views.7/8 without access to TNE felt a TNE service would be beneficial to their trust and 5 would be keen to set it up in their trust. Reported barriers to set up were cost $6 / 8$ and time 1/8. 6 would be more likely to set up a TNE service if training were available.

Conclusion TNE is not widely used in our region with only 1 of 10 trusts performing regular TNE lists. It is perceived by the majority of endoscopists to have significant patient benefit and the majority are keen to set up a service. The main restriction to use appears to be the cost of set up despite the opinion that TNE is cost efficient overall. It is indicated that making TNE training available may increase its use. This was a regional survey and it would be interesting to see if these results are replicated nationally.

Disclosure of Interest C. Parker Grant/Research Support from: Submitting author's post is funded by Imotech Medical, S. Panter: None Declared

\section{PTH-135 EXAMINING THE ATTITUDES, PERCEPTIONS AND BARRIERS OF BOWEL SCREENING WALES STAKEHOLDERS}

doi:10.1136/gutjnl-2013-304907.622

1."D Snelling, 'H Heard. 'Screening Division, Public Health UK NHS Trust, Cardiff, UK

Introduction Service evaluation is of paramount importance for the continued improvement and development of any health intervention and there is very little documented evidence that examines the attitudes and perceptions of Bowel Screeing Wales (BSW) stakeholders. Anecdotal evidence has suggested several factors that contribute towards the uptake of bowel screening in Wales, such as, lack of understanding around screening, the nature of the test, and the will to complete the test, but this evidence has not been evaluated or documented.

Methods A qualitative descriptive approach was undertaken and semi-structured interviews were conducted with stakeholders at the Royal Welsh Show, Builth Wells, Powys during July 2012 to gauge their attitudes, perceptions, and barriers towards bowel screening. Inclusion criteria was for all eligible men and women aged between 60-74 years who have been invited to be screened.

Results 42 participants agreed to take part in the interview (19 male and 23 female) of which 31 participants reported completing their bowel screening test with 12 participants reporting they had not.

The results indicate that participants are aware of cancer and have a very basic knowledge regarding bowel cancer but are not necessarily aware of the function of the bowel screening programme. A content analysis framework was developed (Newell \& Burnard, 2006) which identified two major themes; health beliefs and health behaviour. This service evaluation suggests that participant's perceived susceptibility influences their decisions to take part. Participants who are not aware of BSW or the risks associated with bowel cancer will not complete the kit. Furthermore, this service evaluation suggests that participants who do not present with symptoms are also less-likely to complete their kit. Furthermore, only a very small number of participants sited fear or anxiety as a contributing factor for participating even though they were aware that the kit was to test for cancer. Majority of the participants who declined the invitation suggested that this was due to dealing with their faecal matter. It is interesting to note that their reasons for not completing their kit were lethargy and apathy.

Conclusion Service evaluations are essential in understanding the attitudes and perceptions of stakeholders. The findings from this service evaluation suggest that participants have a limited knowledge of the risks associated with bowel cancer and know very little about the programme but perceive screening to be important. However participants perceived severity and susceptibility are contributing factors in their participation to accept or decline the invitation to be screened.

Disclosure of Interest D. Snelling Employee of: Bowel Screening Wales, H. Heard: None Declared

\section{REFERENCE}

Newell, R; Burnard, P (2006) Research for evidence-based practise. Blackwell publishing. Oxford. UK.

\section{PTH-136 FAECAL CALPROTECTIN - IS IT REQUESTED APPROPRIATELY AND IS IT COST EFFECTIVE?}

doi:10.1136/gutjnl-2013-304907.623

1, ${ }^{*}$ E Derbyshire, ${ }^{2} \mathrm{C}$ Wells, ${ }^{2} \mathrm{~J}$ Hancock, ${ }^{2} \mathrm{D}$ Dwarakanath. 'Gastroenterology, North Cumbria University Hospitals NHS Trust, Carlisle, ' Gastroenterology, North Tees \& Hartlepool NHS Foundation Trust, Stockton-on-Tees, UK

Introduction Faecal calprotectin is a neutrophil derived protein that can be detected by quantitative enzyme linked immunosorbant assay in stool samples. It reliably predicts the level of mucosal inflammation in the lower gastrointestinal tract (1). The high negative predictive value of faecal calprotectin should lead to invasive investigation being avoided with a subsequent reduction in cost and demand on already stretched endoscopy services (2). We reviewed the use of faecal calprotectin in the trust to establish if current practise confirms this.

Methods The hospital numbers of all patients who had a faecal calprotectin processed at North Tees \& Hartlepool NHS Foundation Trust from 01/04/2011 to 31/12/2011 were collected from the biochemistry department's database. These patients' case notes were then analysed to record the indication for faecal calprotectin, its result, subsequent investigation and management.

Results Faecal calprotectin was requested in 2 groups of patients: 68 with lower gastrointestinal symptoms and 44 with Inflammatory Bowel Disease (IBD). Of the 68 patients with lower gastrointestinal symptoms, faecal calprotectin was normal in 48 patients: 13 ( 9 males, 4 females, median age 30 years, commonest symptom abdominal pain) at initial presentation had no further investigation, 23 (11 males, 12 females, median age 41, commonest symptom abdominal pain) went onto have further investigations (12 colonoscopies, 3 flexible sigmoidoscopies and 1 small bowel meal and follow through; all normal); in 12 (3 males, 9 females, median age 45 , commonest symptom chronic diarrhoea) the negative faecal calprotectin was as a second line following initial investigation including 8 normal colonoscopies and 1 normal flexible sigmoidoscopy. Of those patients with IBD, faecal calprotectin was normal in 9 patients. In 7 patients management decisions were taken on the basis of its result alone. These included commencement of Infliximab $(n=2)$, 6-mercaptopurine $(n=1)$, azathoprine $(n=1)$, pentasa $(\mathrm{n}=2)$ and prednisolone $(\mathrm{n}=1)$ without further investigation. 
Conclusion A negative faecal calprotectin led directly to the avoidance of a colonoscopy in 12 patients and of small bowel investigation in 11 patients. Given the trust's current tariffs for faecal calprotectin, colonoscopy and small bowel meal and follow through, a total cost saving of $£ 7,194.59$ was made. Avoiding further investigation by waiting for a negative faecal calprotectin would have resulted in a greater cost saving.

Disclosure of Interest None Declared.

\section{REFERENCES}

1. CA Lamb, JC Mansfield. Measurement of faecal calprotectin and lactoferrin in inflammatory bowel disease. Frontline Gastroenterol 2011; 2:13-18.

2. James Turvill. High negative predictive value of a normal faecal calprotectin in patients with symptomatic intestinal disease. Frontline Gastroenterol 2012; 3:21-28.

\section{PTH-137 PATIENTS' VIEWS ON THEIR EXPERIENCE OF THE DELIVERY OF SINGLE-SEX ACCOMMODATION WITHIN THE ENDOSCOPY DEPARTMENT: IS IT WORTH IT?}

doi:10.1136/gutjnl-2013-304907.624

1."E F Wiseman, 'S Shah, 'Y S Ang, 'R R Keld. 'Gastroenterology, Royal Albert Edward Infirmary, Wigan, UK

Introduction The 2007 Chief Nursing Officer's report on privacy and dignity identified provision of single-sex accommodation (SSA) as a key objective for the NHS. This was formalised in the 2010 Department of Health (DOH) policy to eliminate mixed-sex accommodation and financial sanctions for policy breaches were introduced in 2011. Our endoscopy department adopted the policy in 2011 . However the unit, which opened in 2004, has only one recovery bay, necessitating separate gender lists. Urgent procedures for patients of the opposite sex to the list running are accommodated by admission/recovery in a separate endoscopy room. We explored the views of patients on their experience of attending our unit since implementation of the SSA policy. There are no published studies of patients' perspectives of care in endoscopy units since the widespread adoption of the policy in 2011

Methods Patients attending the endoscopy unit between August and October 2012 were invited to take part in the study by nursing staff during the admission process. Patient views were assessed using a structured non-disguised questionnaire of ten closed-ended questions. The Student's $t$-test was used and a p value of $<0.05$ was taken to be significant.

Results Of the 68 questionnaires returned (female 20, male 25, unknown 23) 14 (20.6\% [80\% female]) and 17 (25\% [81.8\% female]) reported that they would feel vulnerable changing behind a curtain or waiting in a gown in a mixed-sex area respectively. Patients ranked (scale 1-10, 1 = least, $10=$ most) the importance of provision of SSA significantly lower than the importance of access to prompt investigation and treatment (mean: 4.8 [SD \pm 3.74$]$ vs 8.71 [SD \pm $\left.2.70], p=2.6 \times 10^{-7}\right)$. Male patients ranked the importance of SSA significantly lower than females (mean:1.5 [SD \pm 1.05$]$ vs 6.5 [SD $\left.\pm 3.30], p=6.3 \times 10^{-6}\right) .17 / 68$ patients $(25 \%)$ were admitted to an area other than the main receiving/recovery area because they were a different sex to the list running, and of these, 7/17 (41.2\%) felt their care was compromised or patient experience reduced as a result.

Conclusion SSA delivery is important to our patients, especially women. However they rank prompt investigation and treatment as more important. The rapid introduction of SSA in our hospital, in the absence of the necessary infrastructure, conflicts in part, with the pressure to deliver timely investigations. This can lead to compromised care, notably in patients who are admitted/recovered in an alternative room and can also lead to delays for specialised endoscopy (polypectomy, ERCP and EUS). By making such compromises we are at risk of achieving no net gain in patient satisfaction and experience.

Disclosure of Interest None Declared.

\section{PTH-138 ASSESSING THE POSITIVE PREDICTIVE VALUE OF PEPTIC ULCERATION ON ENDOSCOPY FOR THE DIAGNOSIS OF HELICOBACTER IN A GENERAL POPULATION}

doi:10.1136/gutjnl-2013-304907.625

1. ${ }^{*} \mathrm{~F}$ K Shaikh, 'A A Palejwala, 'M Madan. 'Gastroenterology/General Medicine, Burton Hospitals Foundation NHS Trust, Burton On Trent, UK

Introduction Helicobacter Pylori (H. Pylori) is a gramme negative bacillus. It is strongly associated with peptic ulcer disease and gastric cancer. $50 \%$ of the population aged over 50 may be infected with H. Pylori: The prevalence in 2008 was $30-40 \%$ in the UK adult population with pockets of higher prevalence associated with deprivation. Different diagnostic tests including ${ }^{13} \mathrm{C}$ urea breath test, stool antigen test, serum antibodies to $\mathrm{H}$. pylori and rapid urease (CLO) test are commonly used in current medical practise. Histological detection of $\mathrm{H}$. Pylori in gastric biopsy specimens still remains the gold standard investigation for diagnostic purposes. Our study was to assess whether peptic ulceration at endoscopy should be used to determine Helicobacter testing or whether all patient referred for Gastroscopy with ' non-reflux dyspepsia' should be offered testing.

Methods It was a retrospective observational study analysing results of consecutive 172 patients who had CLO test performed (male 89, female 83) on a single user operator endoscopy list over a 4 months period (March to June 2010). CLO testing was carried out on the discretion of the endoscopist on any patient with unexplained 'dyspepsia' or endoscopic findings of peptic ulceration. Data on whether patients were on a proton pump inhibitor at the time of the endoscopy or concurrent use of non-steroidal anti-inflammatory drugs (NSAIDS) was not recorded.

Results Out of 172 cases, 34 cases were tested CLO positive (12/34 CLO positive patients had evidence of peptic ulcer disease on OGD).138 cases were tested CLO negative of which 62/138 had evidence of peptic ulcer disease. Prevalence figure in our study matched with national UK figures i.e $43.02 \%$ (95\% CI: $35.51 \%$ to $50.78 \%$ ).

Conclusion Approximately $1 / 3$ of patients found to be CLO positive had signs of peptic ulceration (35\%). In the same cohort of patients nearly $1 / 2$ of patients found to be CLO negative also had signs of peptic ulceration (45\%). In our study using evidence of peptic ulceration (gastritis, duodenitis, gastric and duodenal erosions/ ulcers) as a guide as to whether a CLO test should be carried out is unhelpful. Caution has to be taken as we did not take into account data as to usage of PPI or NSAIDS. We suggest that presence of endoscopic findings should not be a sole determinant for Helicobacter testing.

Disclosure of Interest None Declared.

\section{PTH-139 A UNIOUE COMBINED GASTROENTEROLOGY/ RHEUMATOLOGY CLINIC: THE FIRST YEAR}

doi:10.1136/gutjnl-2013-304907.626

${ }^{1} \mathrm{G}$ Constable, ${ }^{2, *} \mathrm{~F}$ Bari, ${ }^{2} \mathrm{~T}$ Lawson. ${ }^{1}$ Gastroenterology; ${ }^{2}$ Rheumatology, Princess of UK Hospital, Bridgend, UK

Introduction Articular problems affect many patients with inflammatory bowel disease (IBD) and joint symptoms are often difficult to control despite the therapeutic strategies aimed at controlling gut inflammation (1). Patients with inflammatory rheumatic conditions present a range of clinical problems to the gastroenterologist such as IBD, dysmotility, dysbiosis, liver dysfunction, nutritional problems and drug side effects. Patients often drift between the two specialties with inefficient communication and subsequent delay in a joined up approach to management. We therefore developed a joint gastroenterology/rheumatology clinic to improve the care of these complex patients and now report our experience of the first year. 